\title{
RSA, TSA and PyC hemi-prostheses: comparing indications and clinical outcomes using a second-generation modular short-stem shoulder prosthesis
}

\author{
Benjamin D. Kleim ${ }^{1,2} \cdot$ Christina Garving $^{1}$ Ulrich H. Brunner ${ }^{1}$
}

Received: 27 January 2020 / Accepted: 14 July 2020 / Published online: 6 October 2020

(c) The Author(s) 2020

\begin{abstract}
Introduction The goal of this study was to provide an insight into the clinical results after modular short-stem shoulder arthroplasty for various indications.

Materials and methods A consecutive cohort study of 76 patients followed up for 23-55 (mean 31.4) months. 23 anatomical (TSA), 32 reverse (RSA) and 21 hemi-prostheses with a pyrocarbon head (PyC), using a modular short stem with proximal porous coating were implanted. Range of motion, pain and Constant score (CS) were recorded. Comparisons of pre- vs postoperative outcomes, between prosthesis types and indications, were made.

Results All prosthesis types brought about a significant improvement $(p<0.05)$ in all measured outcomes. TSA had a significantly higher increase in the CS than PyC and RSA ( $p=0.002$ and 0.003 , respectively). TSA produced superior gains in all ROM compared with RSA $(p<0.02)$. RSA brought about significantly smaller improvements in internal rotation than TSA and PyC $(p=0.0001$ and 0.008 , respectively). TSA had greater pain relief than PyC $(p=0.02)$. TSA with Walch A glenoids seemed to improve more than type B in the CS. PyC patients with Walch B glenoids improved more than Walch A $(p=0.03)$. When implanted due to Osteoarthritis (OA), PyC had a comparable final outcome to TSA $(p=0.95)$, although the preoperatively worse TSA patients had a greater improvement in the CS $(p=0.026)$. The outcome of RSA did not differ between indications, but Walch A glenoids tended to improve more.

Conclusions Using a second-generation short-stem shoulder prostheses, TSA achieves the best clinical improvements overall, especially for OA with a Walch A glenoid. Despite refixation of the subscapularis tendon in all cases, RSA has inferior internal rotation than TSA and PyC, suggesting a mechanical limitation. OA, a Walch B glenoid and arthritis caused by instability seem to be ideal indications when considering PyC.
\end{abstract}

Keywords Short stem $\cdot$ Shoulder arthroplasty $\cdot$ Pyrocarbon $\cdot$ Clinical $\cdot$ Hemiarthroplasty $\cdot$ Modular

\section{Introduction}

Shoulder arthroplasty is an increasingly common therapy for osteoarthritis, rheumatoid arthritis, cuff tear arthropathy, osteonecrosis as well as intra-articular fractures of the proximal humerus [1,2].

Benjamin D. Kleim

Drkleim@doctors.org.uk

1 Teaching Hospital of the Ludwig-Maximilians-University Munich, Munich, Germany

2 Present Address: Department of Sports Orthopaedic Surgery, Klinikum rechts der Isar, Technical University Munich, Ismaningerstr 22, 81675 Munich, Germany
Uncemented modular short-stemmed prostheses are still a relatively novel design type in shoulder prostheses and early results have been very positive with good function and low complication rates [3, 4]. A benefit is that one stem can be used in different configurations, as part of a hemi-prosthesis, RSA or anatomical TSA.

Hemi-prostheses are often considered for young patients with predominantly humeral disease, to avoid the complications of a glenoid replacement, subsequent bone loss and difficult revision surgery. However, a major problem when replacing only the humeral joint surface, traditionally with a cobalt-chrome head, is progressive glenoid wear and pain $[5,6]$. Consequently, it has been found that TSA has a better outcome and more pain relief than hemiarthroplasty [7]. Pyrocarbon is a novel material thought to have 
biomechanical properties similar to cartilage and is therefore being used in hemi-prostheses in a hope to ameliorate this problem [8]. Clinical results achieved with this new material are yet scarce and to our knowledge no data exist where these are compared to total shoulder replacement.

A recent comparison of elderly patients receiving RSA or TSA for glenohumeral arthritis with an intact rotator cuff was unable to find a significant difference in outcomes [9]. However, other studies have shown TSA to be superior in external rotation [10] and in internal rotation when compared in patients who had contralateral implantation of both RSA and TSA [11].

For cuff tear arthropathy there is a consensus that joint replacement should be performed with RSA. However, for primary osteoarthritis a TSA, RSA or hemi-prosthesis may be used. The choice of which type to implant is based on the patient age, function and disease morphology. The morphology of glenoid wear, as described by Walch and later modified by Bercik [12], has been shown to impact on the outcomes after shoulder arthroplasty. Outcomes of hemiprostheses were found to be adversely affected by eccentric posterior wear [13]. This trend has also been described for outcomes after TSA, although these still had better results than hemi-prostheses in patients with Walch B2 glenoids [14]. For this reason a trend has emerged to opt for RSA in cases with excessive posterior glenoid wear [15].

The aim of this study was to investigate and compare the clinical outcomes of this second-generation short-stem modular shoulder prosthesis, when used in its different forms (TSA, RSA and PyC) and for different indications.

\section{Materials and methods}

\section{Patient population and study design}

In this single-centre cohort study, all 103 patients who consecutively underwent shoulder arthroplasty, using a curved titanium short-stem uncemented modular prostheses with a proximal porous coating (Aequalis Ascend Flex ${ }^{\mathrm{TM}}$, Wright Medical, Bloomington, USA), between May 2013 and June 2015 at Agatharied hospital, were invited for follow-up at regular intervals. All the operations were carried out by one of two senior surgeons.

Preoperatively the glenoid retroversion was calculated relative to the Friedman line [16], the inclination according to the Maurer angle [17] using X-rays and CT. The prostheses were implanted in three forms: Hemiarthroplasty using a pyrocarbon head (PyC), anatomic total shoulder prosthesis (TSA) and reversed shoulder prosthesis (RSA). Patients were offered prosthesis types best suited to their pathology: Patients with primary osteoarthritis, intact rotator cuffs, a glenoid retroversion of $<10^{\circ}$ and a posterior subluxation of the humeral head of $<80 \%$ underwent anatomical TSA; patients with little or no glenoid pathology, an intact rotator cuff and younger age were offered a PyC hemiprosthesis; lastly patients with rotator cuff pathology, glenohumeral subluxation of $>80 \%$ or a glenoid retroversion of $10^{\circ}$ or more were treated with RSA. If the retroversion was $>10^{\circ}$, this was corrected with the use of autologous wedge-shaped cancellous bone grafting (wedged BIO-RSA) under the base plate ( 4 cases). Furthermore, in 2 cases with extreme glenoid wear, BIO-RSA was employed to lateralize the base plate. The subscapularis tendon was repaired in all cases transosseously in double-row technique.

10 Patients were excluded from this study: 1 with a hemiprosthesis with a titanium head (in place of the pyrocarbon due to nickel allergy) and 9 with complications as outlined in the results below, leaving a potential study group of 93 . Data from preoperative examinations as well as at most recent follow-up were gathered and analysed. The glenoid morphology was described according to the modified Walch classification [12] from the preoperative CT. We had followup data for 76 of the eligible 93 patients (82\%). Patients were lost to follow-up for reasons, such as old age and frailty, death (unrelated to the operation or prosthesis), missing data or refusal of follow-up examinations.

\section{Clinical evaluation}

Preoperatively and during follow-up appointments clinical outcomes, such as range of movement (ROM) and pain on the visual analogue scale (VAS), as well as the validated Constant Score (CS) [18], were recorded. This and further patient information including the demographics, diagnosis and operations were gathered from the patient records. To quantify internal rotation, this was scored as shown in Table 1.

\section{Statistics}

The statistics software SPSS V25.0 (IBM) was used. To assess the significance of changes in pre- and post-operative outcomes, the paired $t$ test was calculated; to compare the delta values (difference between pre- and postoperative)

Table 1 Scoring system for internal rotation

\begin{tabular}{ll}
\hline $\begin{array}{l}\text { Internal rota- } \\
\text { tion score }\end{array}$ & $\begin{array}{l}\text { Level } \\
\text { reached with the } \\
\text { back of the hand }\end{array}$ \\
\hline 0 & Thigh \\
1 & Gluteal \\
2 & Iliosacral joint \\
3 & Lumbar spine \\
4 & Thoracic spine \\
5 & Scapula \\
\hline
\end{tabular}


Table 2 Patient demographics and preoperative diagnoses

\begin{tabular}{lllll}
\hline & Total & TSA & RSA & PyC \\
\hline Number & 76 & 23 & 32 & 21 \\
Age & $68.5(22-84)$ & $70.0(58-84)$ & $74.1(65-84)$ & $58.3(22-84)$ \\
Sex & 45 female & 15 female & 26 female & 4 female \\
Follow-up (months) & $31.4(23-55)$ & $31.6(23-51)$ & $34.3(23-55)$ & $26.7(23-38)$ \\
Primary osteoarthritis & 44 & 23 & 6 & 14 \\
Cuff tear arthropathy & 24 & 0 & 25 & 0 \\
Irreparable rotator cuff tear & 1 & 0 & 1 & 0 \\
Fracture sequelae & 1 & 0 & 0 & 1 \\
Avascular necrosis & 3 & 0 & 0 & 3 \\
Arthritis resulting from instability & 3 & 0 & 0 & 3 \\
\hline
\end{tabular}

Table 3 Preoperative glenoid morphology according to the modified Walch classification [18]

\begin{tabular}{lrrrl}
\hline $\begin{array}{l}\text { Glenoid type } \\
\text { Walch) }\end{array}$ & Total & TSA & RSA & PyC Hemi \\
\hline A1 & 14 & 0 & 9 & 5 \\
A2 & 28 & 13 & 11 & 4 \\
B1 & 19 & 6 & 7 & 6 \\
B2 & 11 & 4 & 2 & 5 \\
B3 & 0 & 0 & 0 & 0 \\
C & 0 & 0 & 0 & 0 \\
D & 4 & 0 & 3 & 1 \\
\hline
\end{tabular}

between implant types, the $t$ test was employed. In each case the significance threshold was set at $p<0.05$.

\section{Results}

\section{Patient demographics}

The patient demographics and diagnoses leading to surgery of the study group of 76 patients are shown in Table 2. With the exception of the PyC subgroup, the cohort was made up of more women than men. The PyC contingent was younger and male dominated. The average age was the highest in the RSA subgroup.
Table 3 shows the glenoid morphologies of the patients prior to surgery. TSA patients had mostly A2 glenoids, but also B1 and B2 wear patterns. RSA also had predominantly A type glenoids, but also B and D morphologies. The PyC subgroup contained patients with mixed glenoid types.

\section{Pre- vs postoperative clinical outcomes}

The clinical outcomes pre- vs postoperative are displayed in Table 4. All patient groups improved significantly $(p<0.05)$ in all outcomes measured. TSA patients had, on average, the lowest preoperative and the highest postoperative CS. The PyC patients had the highest preoperative CS. All patient groups benefited from pain reduction, reducing from VAS 6.6 pre-change to preoperatively down to 1.0 postoperatively. Abduction increased from $88^{\circ}$ to $129^{\circ}$, forward flexion from $93^{\circ}$ to $137^{\circ}$. Internal rotation increased from reaching the gluteal area before, to placing the back of the hand on the lumbar spine after the operation. On average, patients also benefited from more external rotation, $21^{\circ}$ preoperatively and $40^{\circ}$ postoperatively.

\section{Comparison between implant types}

To reduce the confounding effect of the demographical differences in the subgroups, delta values (difference between

Table 4 Pre- and postoperative outcomes for the measured variables for the cohort and subgroups

\begin{tabular}{lccccrrrr}
\hline Outcome & Total pre-OP & Total post-OP & TSA pre-OP & TSA post-OP & RSA pre-OP & RSA post-OP & PyC pre-OP & PyC post-OP \\
\hline CS & 38.2 & 78.3 & 34.5 & 84.0 & 36.9 & 72.9 & 44.2 & 80.3 \\
Pain VAS & 6.6 & 1.0 & 6.7 & 0.37 & 6.8 & 1.1 & 6.4 & 1.5 \\
Abduction $^{\circ}$ & 88.2 & 129.4 & 84.3 & 138.3 & 88.1 & 121.7 & 92.6 & 131.4 \\
Flexion $^{\circ}$ & 92.5 & 137.1 & 87.6 & 144.3 & 91.4 & 128.0 & 99.5 & 143.1 \\
Internal rotation (score) $^{\circ}$ & 1.4 & 2.6 & 1.0 & 3.1 & 1.7 & 2.3 & 1.2 & 2.7 \\
External rotation $^{\circ}$ & 21.1 & 39.6 & 6.1 & 40.0 & 19.8 & 36.3 & 17.6 & 44.3 \\
\hline
\end{tabular}

All comparisons between pre- and postoperative values were statistically significant $(p<0.05)$ 


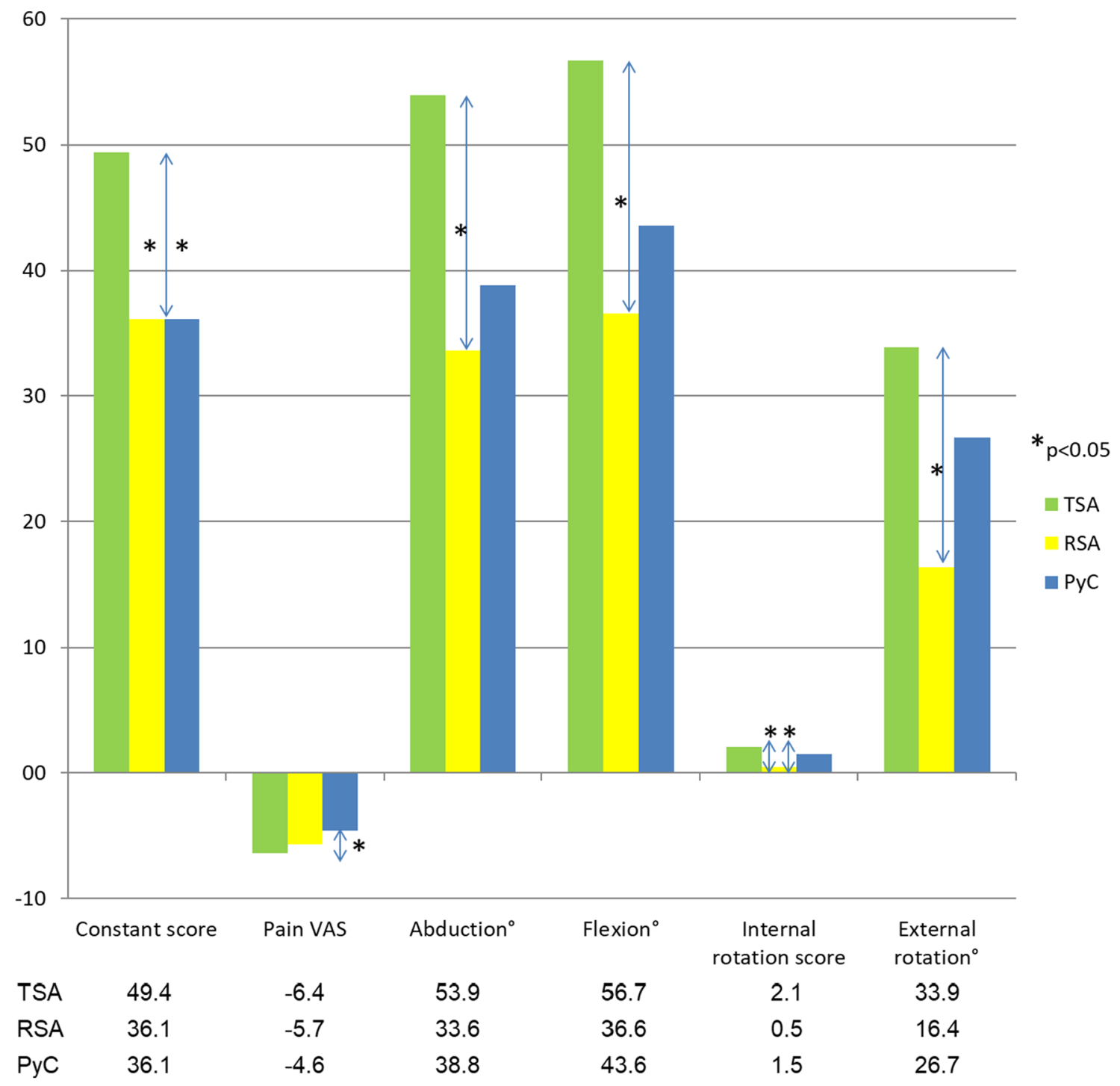

Fig. 1 Delta (postoperative minus preoperative) values for the measured outcomes compared between the implant types. Statistically significant $(p<0.05)$ differences are identified with $\mathrm{a} *$

pre- and postoperative) were compared between prosthesis types (Fig. 1).

Notably, anatomical total shoulder replacements had a significantly higher increase in the CS than the other two types. They also brought about more pain alleviation than the pyrocarbon hemiarthroplasties. In abduction and flexion the RSA had less improvement than the TSA. Both the TSA and the PyC gained more internal rotation than the RSA. Additionally, the TSA had larger improvements in external rotation than the RSA. All other comparisons between the implants were not statistically significant. Nevertheless, the trend emerged that TSA was superior to the other two in all aspects and PyC bettered the RSA in everything but pain reduction.
Given that glenoid morphology is an important predictor of outcome, we investigated the performance of the different prostheses when used in patients of varying glenoid types (see Table 5). Interestingly the final outcome of TSA did not differ between A and B glenoids. However, the improvement achieved with TSA seems to be greater in A than in B glenoid patients, although this was not statistically significant $(p=0.085)$. Conversely, using PyC, patients with B glenoids improved significantly more than those with A glenoids $(p=0.034)$, again achieving the same final outcome. The one patient with a D glenoid in PyC had a poor preoperative function and improved less than A and B patients. When comparing the result with $\mathrm{PyC}$ vs TSA in arthritis with B type glenoids, little 
Table 5 Comparison of mean constant score by prosthesis type and glenoid morphology

\begin{tabular}{llll}
\hline $\begin{array}{l}\text { Glenoid type (modified } \\
\text { Walch) }\end{array}$ & \multicolumn{3}{l}{ Prosthesis type } \\
\cline { 2 - 4 } & TSA & RSA & PyC \\
\hline A & & & \\
Preoperative CS & 28.9 & 30.4 & 52.7 \\
Postoperative CS & 83.5 & 71.5 & 81.7 \\
Delta CS & 53.7 & 41.1 & 29.0 \\
B & & & \\
Preoperative CS & 40.6 & 47.8 & 39.5 \\
Postoperative CS & 84.5 & 76.6 & 82.8 \\
Delta CS & 43.9 & 28.8 & 43.3 \\
D & & & \\
Preoperative CS & - & 49.7 & 18.0 \\
Postoperative CS & - & 74.0 & 39.0 \\
Delta CS & - & 24.3 & 21.0 \\
\hline
\end{tabular}

difference is seen in the outcome or the function gained. In patients with type A glenoids, however, a significantly greater improvement was observed using TSA compared with PyC $(p<0.0001)$. Both subgroups reach a similar postoperative CS.

In the RSA subgroup patients with Walch A glenoids appeared to have the greatest improvement, but this was not significant ( $\mathrm{A}$ vs $\mathrm{B} p=0.091$; A vs $\mathrm{D} p=0.15$ ). The postoperative CS was comparable between all three glenoid types.

Table 6 differentiates the results achieved when applying the different prosthesis types for the various diagnoses. The comparison for TSA and PyC hemi-prostheses in osteoarthritic shoulders revealed a significantly greater improvement in the CS of TSA patients $(p=0.026)$, which had a lower preoperative function. However, there is no difference in the final outcome of the PyC and TSA subgroups $(p=0.95)$. When comparing RSA with TSA in osteoarthritis patients, TSA has a significantly greater increase in the CS $(p=0.011)$ and a better postoperative CS $(p=0.002)$. When comparing the results achieved for OA, there was no significant difference in the delta CS using PyC or RSA $(p=0.43)$. Although PyC seemed to have a better final result, this was not statistically significant $(p=0.058)$.

Comparing the results for different indications within the PyC subgroup, this showed no significant differences in the delta scores or postoperative CS between any diagnoses $(p>0.05)$. However, patients operated for OA and arthritis resulting from instability seemed to have a greater benefit and a better postoperative outcome than those with avascular necrosis and fracture sequelae. Although not statistically significant (or measurable using a $t$ test), the patient with arthritis resulting from a fracture had the worst postoperative
Table 6 Comparison of mean CS achieved using the different prostheses for the various diagnoses

\begin{tabular}{|c|c|c|c|}
\hline \multirow[t]{2}{*}{ Diagnosis } & \multicolumn{3}{|c|}{ Prosthesis type } \\
\hline & TSA & RSA & $\mathrm{PyC}$ \\
\hline \multicolumn{4}{|c|}{ Primary osteoarthritis } \\
\hline Preoperative CS & 34.5 & 42.5 & 45.6 \\
\hline Postoperative CS & 84.0 & 74.7 & 83.8 \\
\hline Delta CS & 49.5 & 32.2 & 38.2 \\
\hline \multicolumn{4}{|l|}{ Cuff tear arthropathy } \\
\hline Preoperative CS & - & 34.8 & - \\
\hline Postoperative CS & - & 72.4 & - \\
\hline Delta CS & - & 37.6 & - \\
\hline \multicolumn{4}{|c|}{ Irreparable rotator cuff tear } \\
\hline Preoperative CS & - & 53 & - \\
\hline Postoperative CS & - & 75 & - \\
\hline Delta CS & - & 22 & - \\
\hline \multicolumn{4}{|l|}{ Fracture sequelae } \\
\hline Preoperative CS & - & - & 50 \\
\hline Postoperative CS & - & - & 61.5 \\
\hline Delta CS & - & - & 11.5 \\
\hline \multicolumn{4}{|l|}{ Avascular necrosis } \\
\hline Preoperative CS & - & - & 41.7 \\
\hline Postoperative CS & - & - & 68 \\
\hline Delta CS & - & - & 26.3 \\
\hline \multicolumn{4}{|c|}{ Arthritis resulting from instability } \\
\hline Preoperative CS & - & - & 38.3 \\
\hline Postoperative CS & - & - & 82.3 \\
\hline Delta CS & - & - & 44 \\
\hline
\end{tabular}

$\mathrm{CS}$ and the least improvement compared to the mean values of the other diagnoses.

In the RSA subgroup patients operated for CTA did not improve significantly differently in the CS $(p=0.53)$, nor did they reach a different outcome according to the CS $(p=0.72)$, when compared with patients with OA. The patient with the irreparable rotator cuff tear, although not statistically testable, also appeared to have an outcome comparable to the other RSA patients.

\section{Complications}

In the study group of 76 patients, 2 developed stress fractures: 1 of the scapular spine, which was successfully operated with an ORIF; one of the acromion, which was treated conservatively. 2 patients were revised with evacuations of postoperative haematomas. There were 2 cases of postoperative anaemia requiring a blood transfusion $(2.7 \%)$ and 4 cases had neurological deficits postoperatively which resolved spontaneously in the months following surgery. 


\section{Complications leading to exclusion}

9 patients had to be excluded as a result of complications which were recorded in the follow-up of the initial patient cohort of 103 shoulder prostheses, as they were no longer deemed comparable to the rest of the cohort: 4 patients were found to have a low-grade infection $(3.88 \%)$ and had to be revised. 2 of these were RSA patients, one of whom was 88 years old, the other was a psoriasis vulgaris patient under methotrexate therapy. TSA and PyC had one case of infection each; in the case of the $\mathrm{PyC}$ this was a patient who had had a previous operation due to a tubercular fracture. 2 of the 4 infections were caused by Cutibacterium acnes (formerly known as Propionibacterium acnes). One patient with a TSA developed a rotator cuff tear and was converted to RSA. There were 2 patients with periprosthetic fractures of the humerus (1.94\%), one of whom was treated at another hospital. The other fell and fractured twice, she was treated conservatively the first time, the second time she was operated with a single cerclage with a good outcome. One RSA patient with extremely osteoporotic bone suffered a bony dislocation of the glenoid component, despite the use of a long peg base plate and 4 screws. This was revised, the glenoid component removed and as a salvage operation a modular exchange to place an anatomical head on the existing shaft was performed. 1 patient underwent a cervical spine operation in the months after her shoulder prosthesis and suffered a neurological deficit ipsilaterally, involving the deltoid, as a complication of this. This resulted in recurrent dislocations of the shoulder prosthesis and a stress fracture of the scapula spine of the operated side, leading to exclusion.

\section{Discussion}

\section{Key results}

This patient cohort showed significant improvements in all measured ROM, pain and the CS compared to preoperative values, using a modular short-stem prosthesis with a proximal porous coating $2-4$ years postoperatively. This correlates with previous findings using first-generation shortstemmed shoulder prostheses $[3,4,19,20]$. The overall very positive clinical outcomes achieved with these shoulder prostheses are also, at least, comparable to those described with the use of standard-stemmed prostheses with diaphyseal anchoring [21, 22].

The modular design of this prosthesis has the advantage of individualized assembly to recreate the anatomy of the proximal Humerus and balance tension of the soft tissues. Eccentric head/tray (depending on anatomical or inverse design) components allow the surgeon to adjust the position of the head/tray by turning it to the ideal position before fixation. In this way ideal coverage without overlap and adjustments of soft tissue tension can be made regardless of the stem position. This is of particular importance in the anatomical prosthesis and may in part have contributed to the successful clinical outcomes.

The hemiprosthesis which was used in this cohort uniformly utilized a novel pyrocarbon head. This material is thought to have a biomechanical profile close to that of cartilage and therefore is hoped to reduce the problem of glenoid wear and pain which complicate traditional hemiarthroplasties [8]. Preliminary results after implantation of these have been encouraging, except in patients with a diagnosis of fracture sequelae (osteonecrosis or secondary osteoarthritis), in a study containing some of the patients from our cohort [23]. Our results equally are encouraging, with improvements in all areas of clinical function. With regard to results in the use for fracture sequelae, we had one patient operated with $\mathrm{PyC}$ for this indication, which yielded a poorer result than all other indications, adding weight to the conclusion drawn by Garret et al. [23].

The RSA used in this cohort has a neck shaft angle (NSA) of $145^{\circ}$. This is an intermediate value between the $135^{\circ}$ and $155^{\circ}$ which inverse prostheses also commonly have. Mechanical studies have shown that a steeper NSA causes earlier impingement in abduction, but reduces glenoid notching and increases joint stability [24]. Computerized models have found a lower NSA $\left(135^{\circ}\right)$ to allow a greater ROM in all motions except abduction [25]. This was also true of internal rotation, although the effect of the lower NSA on internal rotation was negated when the glenosphere had been lateralized. Our cohort demonstrated a successful clinical outcome with this modular tray with a NSA of $145^{\circ}$ in terms of the ROM, with no dislocations, suggesting this may be a good compromise between mobility and stability.

The comparison between the different types of this modular prosthesis was interesting, as it showed several differences in their functional characteristics. Overall, though not always statistically significant, the TSA achieved the best outcomes in all measured variables. Kiet et al. have previously described the outcomes of RSA and TSA to be similar, with only better rotation in the TSA group [10]. They, however, did not compare the delta values of the measured parameters. Contradictory to our findings, Flurin et al. described higher outcome scores in TSA but comparatively greater gains in RSA patients [26]. They had implanted the Equinoxe shoulder platform system (Exactech Inc., Florida). Differences in results compared to our study may be due to the implant or differences in the patient cohorts. Trends emerged that patients with $\mathrm{PyC}$ heads generally had greater improvements in the ROM than RSA, whilst RSA brought 
about more pain relief than PyC. However, the only statistically significant difference between the $\mathrm{PyC}$ and the RSA was that $\mathrm{PyC}$ had greater improvement in internal rotation. This is mirrored by better internal rotation in TSA compared to RSA patients and is a reproducible finding [10, 27]. As we reconstructed the subscapularis tendon in all prosthesis types, this phenomenon may likely be explained as being a result of a mechanical restriction of the RSAs semi-constrained design.

\section{Comparison between glenoid types}

When comparing glenoid types, one important finding was that the final outcome did not differ between TSA patients which had type A or B glenoids. It appears, though, that there may be a greater increase in the CS when operating patients with a centred type A situation. This may be because A glenoids have a purely arthritic problem, which can be solved by replacing the glenoid surface, whereas in the B type situation a soft tissue imbalance complicates the disease. PyC patients with B type glenoids improved more than those with A glenoids, reaching a similar end result. This implies that the presence of a B glenoid does not contraindicate the use of a $\mathrm{PyC}$ hemiprosthesis and rather laments that it may be a good indication. It seems that the additional benefit the A types have over B types in TSA is lost in PyC, as the glenoid is not replaced. Strengthening to this is that patients with B glenoids had comparable outcomes when treated with TSA or PyC, whereas type A glenoids improved significantly more when treated with TSA. This does not entirely fit with the findings of Iannotti, who found that both TSA and hemi-prostheses had worse outcomes in a Walch B2 setting, where TSA was still the better choice [14]. This may be because we grouped all B type glenoids together (roughly equal numbers of B1 and B2), whereas they only looked specifically at B2. Also, they used standard cobaltchrome heads for their hemi-prostheses, so it may be that the new pyrocarbon heads have different clinical properties to these. Perhaps in a type B1 situation with little glenoid wear, the natural glenoid with its labrum is superior to a prosthetic glenoid, giving rise to more clinical improvement when the corresponding arthritic humeral articular surface is replaced. Furthermore, it may be that in a B2 setting with biconcave posterior wear $\mathrm{PyC}$ is more effective than standard cobaltchrome prostheses.

In RSA it appears as if patients with type A glenoids may have the most benefit from the operation. This may be because these are the ones that have cranialized more, rather than posteriorizing and therefore benefit from the distalization of the RSA more. The final outcome appears similar between all glenoid types, including type $\mathrm{D}$, however.

\section{Comparison between Diagnoses}

When comparing outcomes between the different types of prostheses in terms of results achieved for osteoarthritis, these mirrored what we found overall when comparing RSA to the other two types. The comparison between TSA and PyC showed a greater improvement in TSA patients with $\mathrm{OA}$, as was the case in the comparison of the entire subgroups for the prostheses. However, the final outcome of the PyC, when only considering OA patients, now matched that of the TSA patients much more closely. In TSA therefore, older patients with worse preoperative function improve more than the on average younger PyC patients. Nevertheless, the function achieved after 2 years when using PyC for $\mathrm{OA}$ is equal to that of the TSA.

To allow some insight into what a good indication for the novel PyC hemiprostheses may be, it was interesting to try and compare the results achieved for the various diagnoses. Unfortunately, the group sizes were too small to show any significant differences in this regard. It can be said, though, that there is a trend that $\mathrm{OA}$ and arthritis resulting from instability may be the best indications for implanting a PyC hemiarthroplasty.

The improvements and outcomes achieved using RSA seem to be the same irrespective of the preoperative diagnosis. This may be because it has inherent stability due to its semi-constrained design, allows recruitment of the deltoid muscle to substitute rotator cuff function and is limited more by mechanical impingement.

\section{Complications}

The infection rate of $3.88 \%$ documented in our cohort is comparative to findings of a meta-analysis by Zumstein et al. who found an infection rate of 3.8\% in RSA patients [28]. Rates of 1-3.9\% have been described for TSA as well [29], whilst it is thought that rates in RSA patients are higher due to haematoma formation in the increased dead space [30]. This is in line with our results. Differences in the reported infection rates are likely to be a result of follow-up and diagnostic differences. Rates of low-grade infections are often likely to be higher than reported as they are difficult to distinguish from aseptic failure [31]. Cutibacterium acnes (formerly Propionibacterium acnes), which made up half of our 4 cases, has been reported to be present in 18-60\% of infections [30]. It is found in the deep tissues around the shoulder, more commonly in men, when using the deltopectoral approach and it has been found in the joint fluid of $42 \%$ of patients undergoing primary shoulder arthroplasty and more often still in revision surgery [30, 32, 33].

The rate of periprosthetic humeral fractures in our cohort (1.94\%) correlates with 1.6-2.4\% described in the 
literature for standard shaft prostheses [34]. However, we were able to treat a patient with a recurrent periprosthetic fracture successfully with as little as a single cerclage. This may indicate simpler treatment options for periprosthetic fractures around short-stem prostheses, but clearly more data are required to draw any conclusions regarding this. Regarding the two cases of scapula spine and 1 acromion fracture in the potential cohort of 103 patients, it should be noted that these all occurred in RSA patients. This is unlikely to be a coincidence as reverse shoulder prostheses put a lot of stress on the delta muscle which causes tension and can lead to stress fractures of the scapula. This affect may be exacerbated in this model due to the additional lateralization and distalization brought about by the onlay design of the modular tray.

\section{Limitations and generalizability}

The main limitation of this study is the retrospective design and tailored patient selection for the different subgroups, leading to selection bias. The resulting differences in diagnostic indications for arthroplasty and patient demographics in the subgroups limit the extent to which interventions can be reliably compared. Furthermore, the sample size, although when compared with other similar studies in the literature is large, may have inhibited the emergence of further statistical significances in the comparison between the prosthesis's subtypes. The follow-up rate of $82 \%$, which for a mid-term cohort study is satisfactory, could to some extent jeopardize the generalizability of the study. Furthermore, whilst using a single-centre study design increases reliability in a scientific method, it also reduces the extent to which results can be extrapolated to other settings. This must be taken into account when interpreting results. The treatment of this cohort took place before we adopted the use of 3-dimensional planning, so it is not known if and how much correction of version and/or inclination was achieved. Another limitation is that although the subscapularis tendon was repaired in all cases, we did not carry out any controls of the success of this repair.

\section{Conclusions}

This study provides an insight into the clinical properties of the different forms in which modular short-stem shoulder prostheses can be implanted and their results for various indications.

When implanted for the diagnoses for which each were conceptualized, it can be said that TSA patients can expect the greatest clinical improvement postoperatively compared to RSA and PyC. PyC patients seem to have bigger improvements in ROM than RSA patients but may subjectively have less pain reduction. Interestingly, despite refixation of the subscapularis tendon in all prosthesis types, RSA had less improvements in internal rotation than the other two prosthesis types, which suggests a mechanical restriction of RSA.

When comparing the clinical success of these prostheses when used for different glenoid types according to the modified Walch classification, we can surmise that TSA has the same outcome regardless of A or B glenoid type, but possibly with more improvement for type A glenoids. Novel PyC hemiprostheses are a good indication for patients with type A and type B glenoids, with equal clinical outcomes and a greater improvement in B glenoids. In fact, type B glenoids were treated just as successfully with $\mathrm{PyC}$ as with TSA.

If $\mathrm{OA}$ is the indication for arthroplasty, TSA and PyC achieve a comparable clinical result $2-4$ years postoperatively, although the preoperatively worse TSA patients have more improvement. It can be said that if glenoid replacement is to be avoided, for example due to young age, that OA patients or those with arthritis resulting from instability and especially in the presence of a type $\mathrm{B}$ glenoid seem to be good candidates for treatment with PyC.

The diagnosis for which RSA is implanted does not seem to greatly affect the outcome, but those with a Walch A glenoid may improve more.

Acknowledgements The authors would like to thank Dr. med. Rudolf Nadjar (Krankenhaus Agatharied) for the inclusion of his patients in the study.

Funding No funding was sought or received to undertake or publish this study. Open Access funding provided by Projekt DEAL.

\section{Compliance with ethical standards}

Conflict of interest B. D. Kleim: None. C. Garving: None. U. H. Brunner: Consultant for Wright Medical, has given paid presentations for Wright Medical.

Ethical approval Ethical approval was sought and granted by the ethics commission of the medical faculty of the Ludwig-MaximiliansUniversity Munich (Reference number: 667-15) in advance of study commencement.

Open Access This article is licensed under a Creative Commons Attribution 4.0 International License, which permits use, sharing, adaptation, distribution and reproduction in any medium or format, as long as you give appropriate credit to the original author(s) and the source, provide a link to the Creative Commons licence, and indicate if changes were made. The images or other third party material in this article are included in the article's Creative Commons licence, unless indicated otherwise in a credit line to the material. If material is not included in the article's Creative Commons licence and your intended use is not permitted by statutory regulation or exceeds the permitted use, you will need to obtain permission directly from the copyright holder. To view a copy of this licence, visit http://creativecommons.org/licenses/by/4.0/. 


\section{References}

1. Dillon MT, Ake CF, Burke MF, Singh A, Yian EH, Paxton EW, Navarro RA (2015) The Kaiser Permanente shoulder arthroplasty registry: results from 6,336 primary shoulder arthroplasties. Acta Orthop 86(3):286-292. https://doi.org/10.3109/17453 674.2015.1024565

2. Kim SH, Wise BL, Zhang Y, Szabo RM (2011) Increasing incidence of shoulder arthroplasty in the United States. J Bone Joint Surg Am 93(24):2249-2254. https://doi.org/10.2106/jbjs.J.01994

3. Bell S, Coghlan J (2014) Short stem shoulder replacement. Int J Shoulder Surg 8(3):72-75. https://doi.org/10.4103/09736042.140113

4. Schnetzke M, Coda S, Walch G, Loew M (2015) Clinical and radiological results of a cementless short stem shoulder prosthesis at minimum follow-up of two years. Int Orthop 39(7):1351-1357. https://doi.org/10.1007/s00264-015-2770-2

5. Johnson MH, Paxton ES, Green A (2015) Shoulder arthroplasty options in young $(<50$ years old $)$ patients: review of current concepts. J Shoulder Elbow Surg 24(2):317-325. https://doi. org/10.1016/j.jse.2014.09.029

6. Schoch B, Schleck C, Cofield RH, Sperling JW (2015) Shoulder arthroplasty in patients younger than 50 years: minimum 20 -year follow-up. J Shoulder Elbow Surg 24(5):705-710. https://doi. org/10.1016/j.jse.2014.07.016

7. Bryant D, Litchfield R, Sandow M, Gartsman GM, Guyatt G, Kirkley A (2005) A comparison of pain, strength, range of motion, and functional outcomes after hemiarthroplasty and total shoulder arthroplasty in patients with osteoarthritis of the shoulder. A systematic review and meta-analysis. J Bone Joint Surg Am 87(9):1947-1956. https://doi.org/10.2106/jbjs.d.02854

8. Salkeld SL, Patron LP, Lien JC, Cook SD, Jones DG (2016) Biological and functional evaluation of a novel pyrolytic carbon implant for the treatment of focal osteochondral defects in the medial femoral condyle: assessment in a canine model. J Orthop Surg Res 11(1):155. https://doi.org/10.1186/s13018-016-0488-5

9. Wright MA, Keener JD, Chamberlain AM (2020) Comparison of clinical outcomes after anatomic total shoulder arthroplasty and reverse shoulder arthroplasty in patients 70 years and older with glenohumeral osteoarthritis and an intact rotator cuff. J Am Acad Orthop Surg 28(5):e222-e229. https://doi.org/10.5435/jaaos -d-19-00166

10. Kiet TK, Feeley BT, Naimark M, Gajiu T, Hall SL, Chung TT, Ma CB (2015) Outcomes after shoulder replacement: comparison between reverse and anatomic total shoulder arthroplasty. J Shoulder Elbow Surg 24(2):179-185. https://doi.org/10.1016/j. jse.2014.06.039

11. Cox RM, Padegimas EM, Abboud JA, Getz CL, Lazarus MD, Ramsey ML, Williams GR Jr, Horneff JG 3rd (2018) Outcomes of an anatomic total shoulder arthroplasty with a contralateral reverse total shoulder arthroplasty. J Shoulder Elbow Surg 27(6):998-1003. https://doi.org/10.1016/j.jse.2017.12.005

12. Bercik MJ, Kruse K 2nd, Yalizis M, Gauci MO, Chaoui J, Walch G (2016) A modification to the Walch classification of the glenoid in primary glenohumeral osteoarthritis using three-dimensional imaging. J Shoulder Elbow Surg 25(10):1601-1606. https://doi. org/10.1016/j.jse.2016.03.010

13. Levine WN, Djurasovic M, Glasson JM, Pollock RG, Flatow EL, Bigliani LU (1997) Hemiarthroplasty for glenohumeral osteoarthritis: results correlated to degree of glenoid wear. J Shoulder Elbow Surg 6(5):449-454. https://doi.org/10.1016/s1058 -2746(97)70052-1

14. Iannotti JP, Norris TR (2003) Influence of preoperative factors on outcome of shoulder arthroplasty for glenohumeral osteoarthritis. JBJS 85(2):251-258
15. Mizuno N, Denard PJ, Raiss P, Walch G (2013) Reverse total shoulder arthroplasty for primary glenohumeral osteoarthritis in patients with a biconcave glenoid. J Bone Joint Surg Am 95(14):1297-1304. https://doi.org/10.2106/jbjs.L.00820

16. Friedman RJ, Hawthorne KB, Genez BM (1992) The use of computerized tomography in the measurement of glenoid version. $\mathrm{J}$ Bone Joint Surg Am 74(7):1032-1037

17. Maurer A, Fucentese SF, Pfirrmann CW, Wirth SH, Djahangiri A, Jost B, Gerber C (2012) Assessment of glenoid inclination on routine clinical radiographs and computed tomography examinations of the shoulder. J Shoulder Elbow Surg 21(8):1096-1103. https://doi.org/10.1016/j.jse.2011.07.010

18. Constant CR, Murley AH (1987) A clinical method of functional assessment of the shoulder. Clin Orthop Relat Res 214:160-164

19. Giuseffi SA, Streubel P, Sperling J, Sanchez-Sotelo J (2014) Shortstem uncemented primary reverse shoulder arthroplasty: clinical and radiological outcomes. Bone Joint J 96(4):526-529. https:// doi.org/10.1302/0301-620x.96b3.32702

20. Loew M (2013) Short stem shoulder prosthesis: concept and first results. Orthopade 42(7):501-506. https://doi.org/10.1007/s0013 2-012-2021-9

21. Iriberri I, Candrian C, Freehill MT, Raiss P, Boileau P, Walch G (2015) Anatomic shoulder replacement for primary osteoarthritis in patients over 80 years: outcome is as good as in younger patients. Acta Orthop 86(3):298-302. https://doi. org/10.3109/17453674.2015.1006036

22. Boileau PWD, Hatzidakis AM, Hovorka I (2006) Neer Award 2005: the Grammont reverse shoulder prosthesis: results in cuff tear arthritis, fracture sequelae, and revision arthroplasty. J Shoulder Elbow Surg 15(5):527-540. https://doi.org/10.1016/j. jse.2006.01.003

23. Garret J, Harly E, Le Huec JC, Brunner U, Rotini R, Godeneche A (2019) Pyrolytic carbon humeral head in hemi-shoulder arthroplasty: preliminary results at 2-year follow-up. JSES Open Access 3(1):37-42. https://doi.org/10.1016/j.jses.2018.09.002

24. Oh JH, Shin SJ, McGarry MH, Scott JH, Heckmann N, Lee TQ (2014) Biomechanical effects of humeral neck-shaft angle and subscapularis integrity in reverse total shoulder arthroplasty. J Shoulder Elbow Surg 23(8):1091-1098. https://doi.org/10.1016/j. jse.2013.11.003

25. Werner BS, Chaoui J, Walch G (2017) The influence of humeral neck shaft angle and glenoid lateralization on range of motion in reverse shoulder arthroplasty. J Shoulder Elbow Surg 26(10):1726-1731. https://doi.org/10.1016/j.jse.2017.03.032

26. Flurin PH, Marczuk Y, Janout M, Wright TW, Zuckerman J, Roche CP (2013) Comparison of outcomes using anatomic and reverse total shoulder arthroplasty. Bull Hosp Jt Dis 71(Suppl 2):101-107

27. Triplet JJ, Everding NG, Levy JC, Moor MA (2015) Functional internal rotation after shoulder arthroplasty: a comparison of anatomic and reverse shoulder arthroplasty. J Shoulder Elbow Surg 24(6):867-874. https://doi.org/10.1016/j.jse.2014.10.002

28. Zumstein MA, Pinedo M, Old J, Boileau P (2011) Problems, complications, reoperations, and revisions in reverse total shoulder arthroplasty: a systematic review. J Shoulder Elbow Surg 20(1):146-157. https://doi.org/10.1016/j.jse.2010.08.001

29. Bohsali KI, Wirth MA, Rockwood CA Jr (2006) Complications of total shoulder arthroplasty. J Bone Joint Surg Am 88(10):22792292. https://doi.org/10.2106/jbjs.f.00125

30. Elizabeth M, Pinder JCO, Stephen Bale R, Trail Ian A (2016) Ten questions on prosthetic shoulder infection. Shoulder Elbow 8(3):151-157. https://doi.org/10.1177/1758573216632464

31. Trampuz A, Zimmerli W (2005) Prosthetic joint infections: update in diagnosis and treatment. Swiss Med Wkly 135(17-18):243-251

32. Levy O, Iyer S, Atoun E, Peter N, Hous N, Cash D, Musa F, Narvani AA (2013) Propionibacterium acnes: an underestimated 
etiology in the pathogenesis of osteoarthritis? J Shoulder Elbow Surg 22(4):505-511. https://doi.org/10.1016/j.jse.2012.07.007

33. Hudek R, Sommer F, Kerwat M, Abdelkawi AF, Loos F, Gohlke F (2014) Propionibacterium acnes in shoulder surgery: true infection, contamination, or commensal of the deep tissue? J Shoulder Elbow Surg 23(12):1763-1771. https://doi.org/10.1016/j. jse.2014.05.024
34. Greiner S, Stein V, Scheibel M (2011) Periprosthetic humeral fractures after shoulder and elbow arthroplasty. Acta chirurgiae orthopaedicae et traumatologiae Cechoslovaca 78(6):490-500

Publisher's Note Springer Nature remains neutral with regard to jurisdictional claims in published maps and institutional affiliations. 